\title{
Welche winkelsteifen Implantate gibt es? Platten, Nägel, Fixateure
}

\section{Dankward Höntzsch}

\section{Zusammenfassung}

Winkelsteife bzw. winkelstabile Implantate repräsentieren ein mechanisch sehr wertvolles Stabilisierungssystem. Wir finden dies heute bei Platten, Nägeln und Fixateuren. Besonders bei den Platten hat sich das winkelsteife Prinzip sehr rasch verbreitet, bei den Marknägeln ist es im Kommen, bei den Fixateuren ist es systembedingt vorgegeben. Das Prinzip ist grundsätzlich nicht neu. Der klassischste Vertreter sind alle Formen der Winkelplatten, welche früher aber auch heute und sicher in Zukunft zu den wertvollsten Implantaten gehören. Das Hauptaugenmerk richtet sich heute auf die erst bei der Osteosynthese intraoperativ entstehende Winkelsteifigkeit zwischen Platten und Schrauben. Diese Systeme werden damit zu einem Fixateur interne und verlassen damit die Prinzipien der Platte, welche ihre Stabilität durch Anpressdruck und Friktion erzielt. Bei den Marknägeln finden ähnliche Mechanismen bereits Anwendung und werden in Zukunft technologisch weiter verbessert. Die winkelstabilen Systeme bieten biomechanische Vorteile, welche sich in der Praxis (empirisch) bewährt haben. Die wissenschaftliche Beweisführung hat begonnen und zeigt erste Früchte. Weitere Untersuchungen müssen die Verbreitung der winkelstabilen Systeme untermauern. Dies nicht zuletzt deshalb, weil mit den winkelstabilen Systemen im Regelfall höhere Kosten anfallen. Auch ist der Lernprozess noch nicht $\mathrm{zu}$ Ende. Wir werden auch eine neue Form des Implantatversagens finden,

\section{Einleitung}

Winkelstabile Plattensysteme erobern derzeit alle Indikationsbereiche. Winkelstabile Plattensysteme sind derzeit das Paradebeispiel und der „Leistungsträger“ für winkelstabile Systeme. Das winkelstabile Prinzip findet sich derzeit und in $\mathrm{Zu}$ kunft vermehrt auch bei den anderen Osteosynthesesystemen. Dieses Heft wird davon berichten. Ob die winkelstabilen Platten als Platten oder als Fixateur interne bezeichnet werden, ist für den Anwender eine akademische Diskussion, aber die Bezeichnung ,internes Fixationssystem“ deutet auf die völlig differente Biomechanik hin. Diese gilt es zu beachten und die technischen Vorgaben umzusetzen. Hier sind Schulung und Information notwendig. Die winkelstabile
Platte/interner Fixateur ist erfolgreich und viele fragen sich, warum gibt es diese Technologie nicht schon lange? Es soll gezeigt werden, dass es seit langer Zeit winkelstabile Systeme gibt. Das Hauptaugenmerk gilt aber einer Übersicht der heute zur Verfügung stehenden winkelstabilen Systeme.

Winkelstabile Systeme aus der Vergangenheit:

- Fixateur externe

- Winkelplatten

- DHS, DCS

- Proximale Femurnägel

- Shuli (Gewindescheiben für konventionelle Schrauben)

- Thorp-System

- Fixateur interne

- Ventrale Wirbelsäulensysteme

- Plattenfixateur
Neue zusätzliche winkelstabile Systeme

- Winkelstabile Schrauben-Platten-Systeme, d.h. interne Fixateure (LISS, LCP, T-Fix u. a.)

- Winkelstabile Verriegelung bei Marknägeln (Spiralklinge, Litos-Nagelsystem u.a.)

- Fixateur interne für Wirbelsäulen und Extremitäten

- Winkelstabilität in Kombination mit minimalinvasiven Osteosynthesemethoden (eingeschobene Platten u. a.)

\section{Fixateur externe}

Der Fixateur ist grundsätzlich winkelstabil. Die winkelstabilen Verbindungselemente zwischen den Schrauben und dem Längsträger sind der Schlüsselpunkt aller Fixateursysteme von Lambotte über Hofmann I zu Hofmann II, den AO-Systemen und allen anderen Vertretern. Mit dem Fixateur externe kann jede Fraktur und Luxation im Bereich der Röhrenknochen und der Gelenke von Kopf bis Fuß stabilisiert werden (Abb.1).

Den Fixateur externe findet und fand man auch am Stammskelett, dem Becken und der Wirbelsäule (MAGERL) [1] Anwendung.

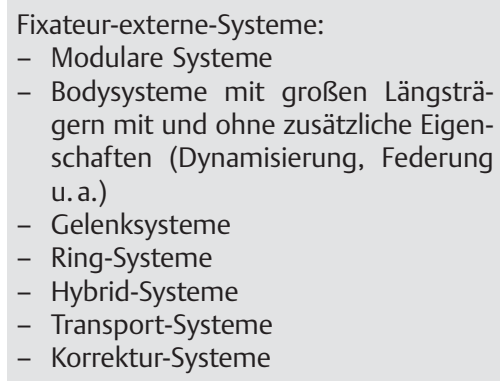
gern mit und ohne zusätzliche Eigenschaften (Dynamisierung, Federung u. a.)

- Gelenksysteme

- Ring-Systeme

- Hybrid-Systeme

- Transport-Systeme

- Korrektur-Systeme

Neben der reinen Stabilisierung findet der Fixateur externe immer mehr Anwendung als intraoperatives Repositionsinstrumentarium (External Reduction Devise - „ERD“) [2]. Im Bereich der Wirbelsäule ist der Fixateur externe als erstes „nach Innen“ gewandert und hat dort den Namen Fixateur interne geprägt. 


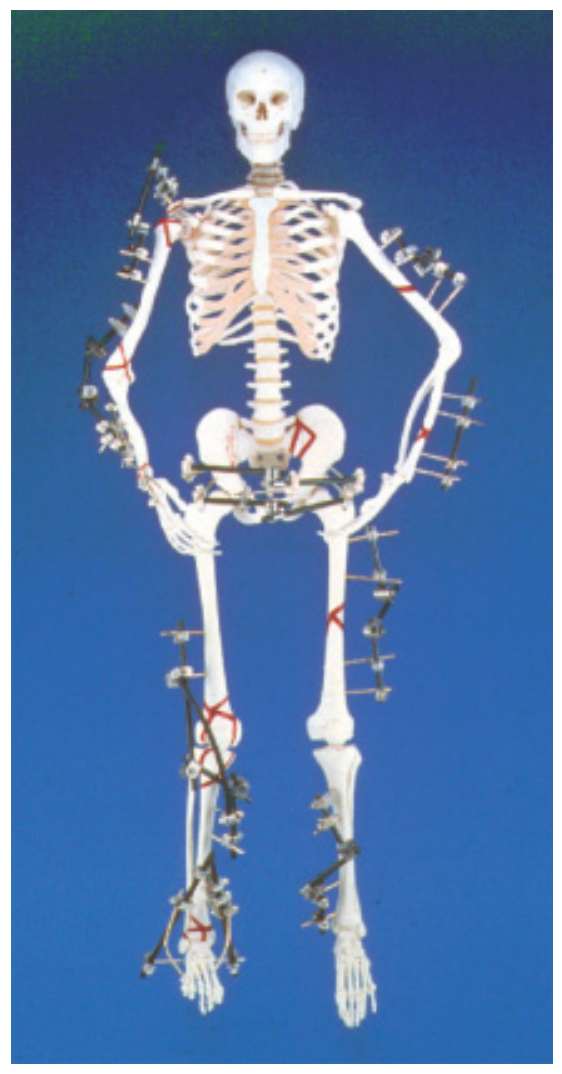

Abb.1 Gelenküberbrückendes und schaftstabilisierendes Fixateur externe „von Kopf bis Fuß".

Somit ist der Fixateur externe der $\mathrm{Na}$ mensgeber für alle internen Fixateure an Wirbelsäule und Extremitäten (Ventrofix, T-fix, Druckplattenfixateur, LISS usw.). Den internen Fixationssystemen steht eine große Zukunft bevor.

\section{Platten/Fixateur interne}

Zur Klassifizierung und Darstellung soll dem Namen Platte der Zusatz interner Fixateur gegeben werden.

\section{Neu? Nein!}

Schon die ersten Plattensysteme von Hausmann waren ein interner Fixateur [4]. Aus „unserer Zeit“ kennen wir als bestes und erfolgreichstes Beispiel für winkelsteife Implantate die Winkelplatten in all ihren Formen und Größen $[1,2]$. Warum waren und sind sie so erfolgreich und wertvoll? Weil sie kleine, schwer zu fassende und/oder zu erreichende (Schenkelhals-)Fragmente stabil fassen und abstützen können, besonders dort, wo das Prinzip Platte mit Anpressdruck und Friktion versagt.

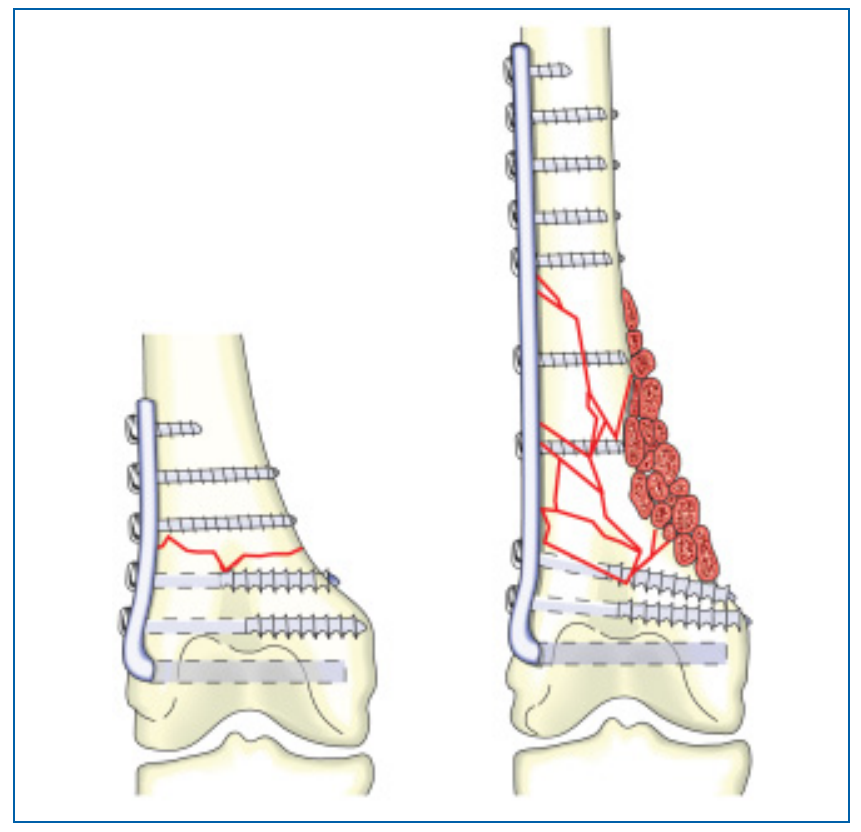

Abb. 2 Die altbewährte und heute noch wertvolle Kondylenplatte.

Winkelstabile Systeme übernehmen in dem Kopfteil von Formplatten z.B. der LISS oder PHILOS-Platte zumindest zum Teil das Prinzip der Winkelplatte (Abb.3). Das Prinzip wird aber nicht nur übernommen, sondern in 2 Punkten verbessert: Das winkelstabile System lässt sich erst intraoperativ „zusammenbauen“. Dadurch entstehen ganz neue Anwendungen und der Stabilisierungscharakter wird durch die Anzahl und Richtung der Schrauben noch wesentlich erhöht. Das bedeutet auch, dass die winkelstabilen Platten wie LISS, PHILOS und distale Radiusplatten bekannte Prinzipien der Winkelplatten übernehmen können. Die Schuli sind gewindetragende
Unterlegscheiben, welche aus konventionellen Kortikalisschrauben und konventionellen Platten eine winkelstabile Schrauben-Platten-Verbindung herstellen (Abb.4) [3]. Es gibt sie seit 10 Jahren, aber sie führen einen Dornröschenschlaf.

Die Idee ist genial, aber für die breite Anwendung zu kompliziert. In Einzelfällen war sie aber sehr hilfreich (Abb.4). Im Wirbelsäulenbereich haben sich winkelstabile Systeme seit langem durchgesetzt. So finden wir diese bei der ventralen Verriegelungsplatte für die Halswirbelsäule oder bei den ventralen Stabilisationssystemen für Brust- und Lendenwirbelsäule. Ein weiterer Vertreter der bewährten

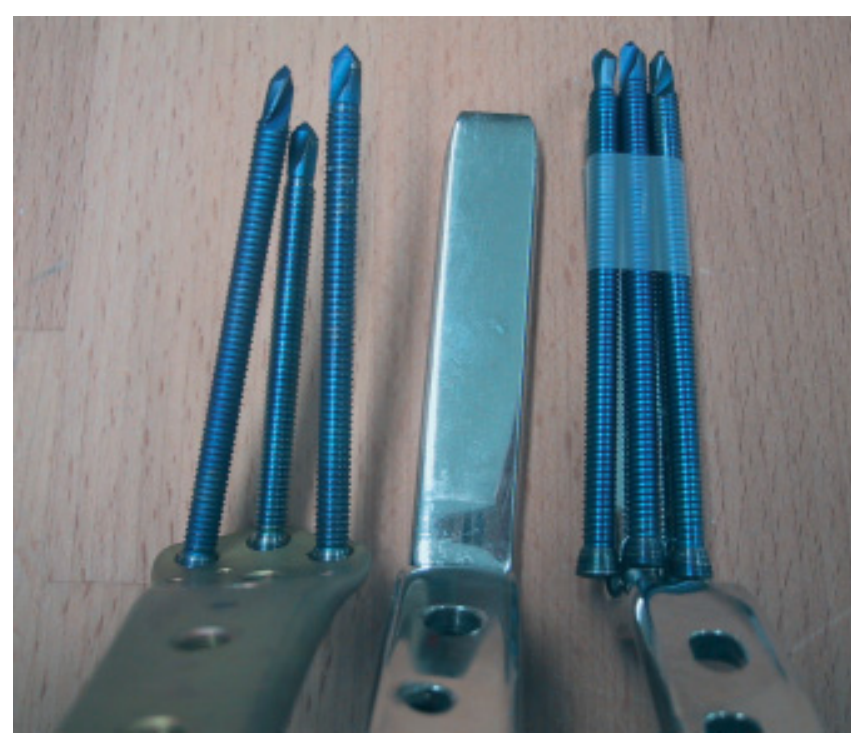

Abb. 3 Drei Schrauben im Kopf einer LISS-Platte haben die gleiche Fläche wie die Klinge einer Kondylenplatte. Hinzu kommt noch die vorteilhafte Spreizung. 


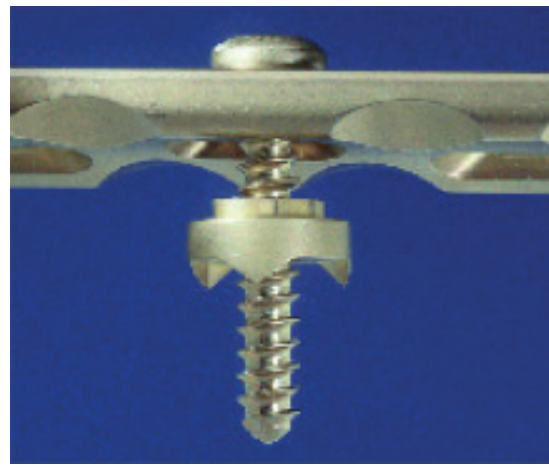

Abb. 4 Mit einer gelenktragenden Unterlegscheibe (Shhuli) wird aus einer konventionellen Platte und konventionellen Schraube ein winkelstabiles System.

und bekannten Systeme ist der Druckplattenfixateur, welcher auch insbesondere an der Wirbelsäule, aber auch an den Extremitäten Anwendung gefunden hat. Für die Kieferchirurgie gibt es seit langem ein winkelstabiles Plattensystem, nämlich das THORP-System der AO. Platten und Schrauben in verschiedenen For-

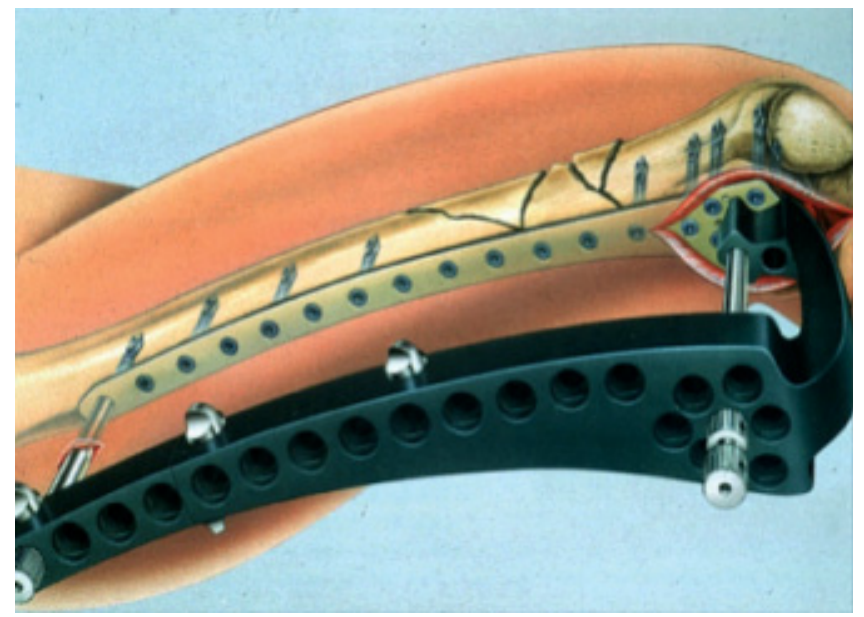

Abb. 5 Low Invasive Stabilization System - LISS. men und Längen sind erhältlich. Die Osteosynthese von schaft- und gelenknahen Frakturen hätte schon lang mit diesem System stabilisiert werden können. Dagegen hat gesprochen, dass die Platten etwas dick sind, etwas teuer und vor allem außerhalb des Horizontes lagen.
Das PC-Fix-System hat die Brücke geschlagen zu den neuen Platten-/Fixateur interne-Systemen. Das PC-Fix-System hat zwei Prinzipien verwirklicht. Nach flächigem Kontakt bei konventionellen Platten wurde über das „Limited Contact Princip“ der nur noch punktförmige Kontakt des Implantats mit dem Knochen

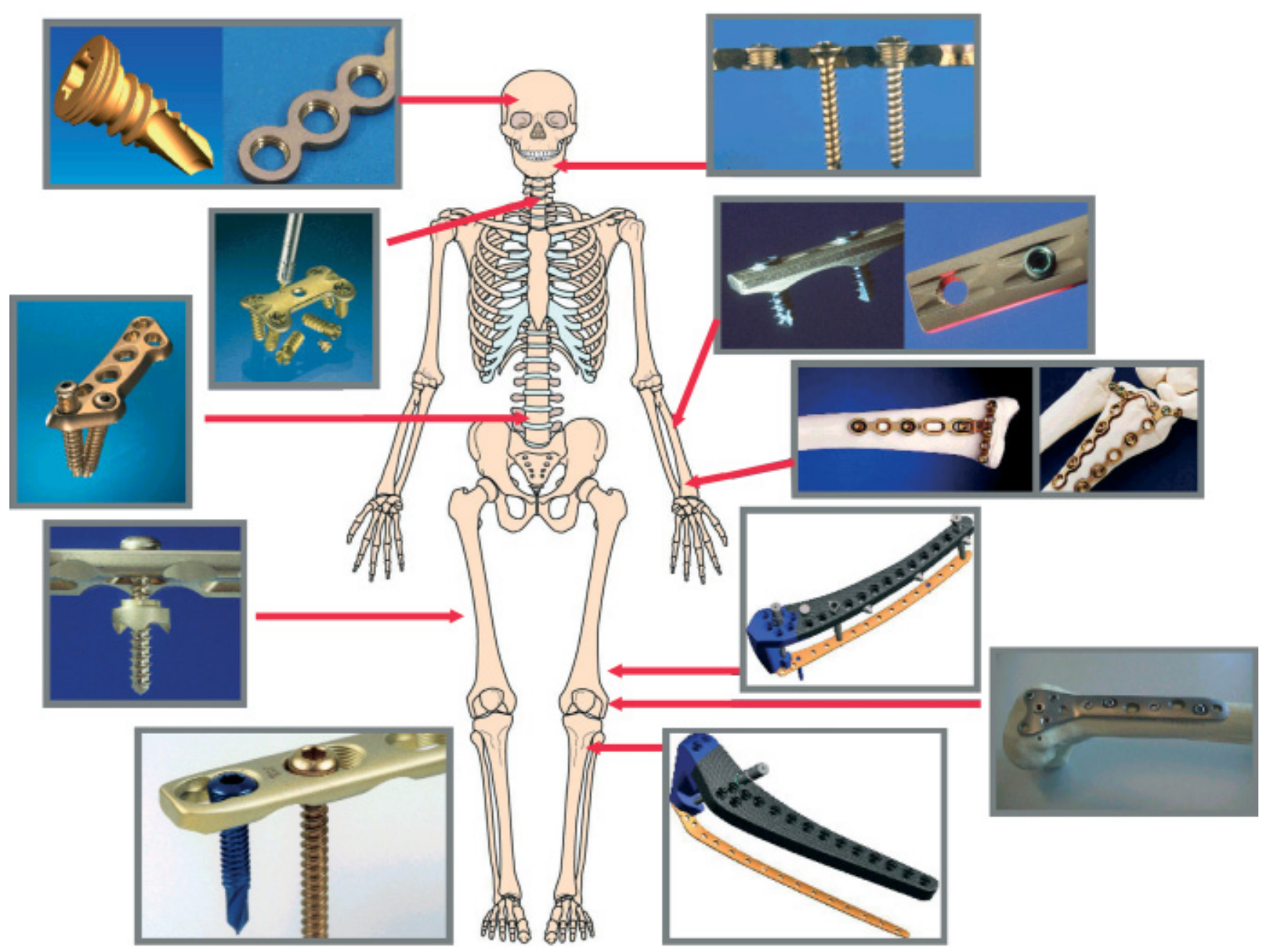

Abb. 6 Verschiedene winkelstabile Systeme nach Robbi Frigg. 

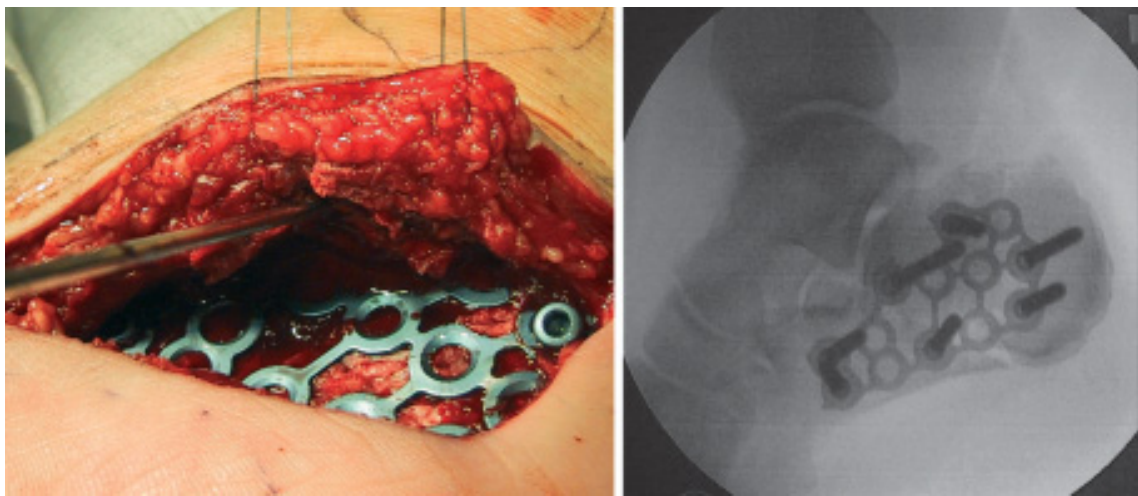

Abb.7 Fersenbeinplatte (LITOS - T-Fix) mit sehr flacher Formgebung und variabeler Winkelfreiheit $\pm 15^{\circ}$.

verwirklicht. Der punktförmige Kontakt diente nur noch zur Abstützung. Punktförmiger Anpressdruck sollte vermieden werden. Deshalb wurde die winkelstabile konische Kopfschraube gewählt. Damit war die Brücke geschlagen zu all den neuen Systemen.

Das „Low Invasive Stabilisation System“ (LISS) hat dann folgende Prinzipien verwirklicht: geeignet für minimalinvasive Methoden zum Einschieben, Winkelstabilität und über dem Periost „schwebend“ (Abb.5). Anwendung findet das System bei den Problemzonen distaler Femur, Femurkondylen, Tibiakopf und proximaler Tibiaschaft.

Heute finden sich bei allen Herstellern gerade Platten und Formplatten mit winkelstabil verankerten Schrauben. Die größten Vorteile werden erzielt bei den gelenknahen Problemfrakturen wie proximaler Humerus, distaler Radius, proximaler und distaler Femur, proximale und distale Tibia sowie Kalkaneus.

Wie wird Winkelstabilität bei Platten/internen Fixateur-Systemen erreicht?

- Durch die Form selbst: Winkelplatte in allen Formen und Größen

- Führung des Schraubenschaftes bei DHS und DCS mit dynamischer Komponente

- Anpressung der Schraubenköpfe: Druckplattenfixateur

- Spreizköpfe: uni- oder aufwändig polyaxial

- Konische Gewindeverbindung: häufigste Lösung

- Gewindeformung: LITOS-System (TFix)

- Mischformen, die beides erlauben, z. B. LCP-System der AO gern bietet die Winkelstabilität Vorteile. Wir finden seit langem bewährte Systeme wie die proximalen Femurmarknägel, neu hinzu genommene Systeme wie Spiralklingen bei UFN und DFN. Zunehmend werden wir auch intramedulläre Kraftträger mit winkelstabil verriegelten Systemen finden, wie es Bolzen oder anders geformte Kraftträger sind.

Winkelstabile intramedulläre Systeme:

- Proximale Femurnägel (mit dynamischer Komponente) (Abb. 8)

- Spiralklingen (DFN, UFN) (Abb. 9)

- Verriegelungsbolzen axial gesperrt (UTN Sonderanfertigung, UHN, DFN, Sirius-System und proximale Humerusnägel)

- Winkelstabil verriegelte Bolzennagelsysteme (in Entwicklung z.B. LITOSSystem)

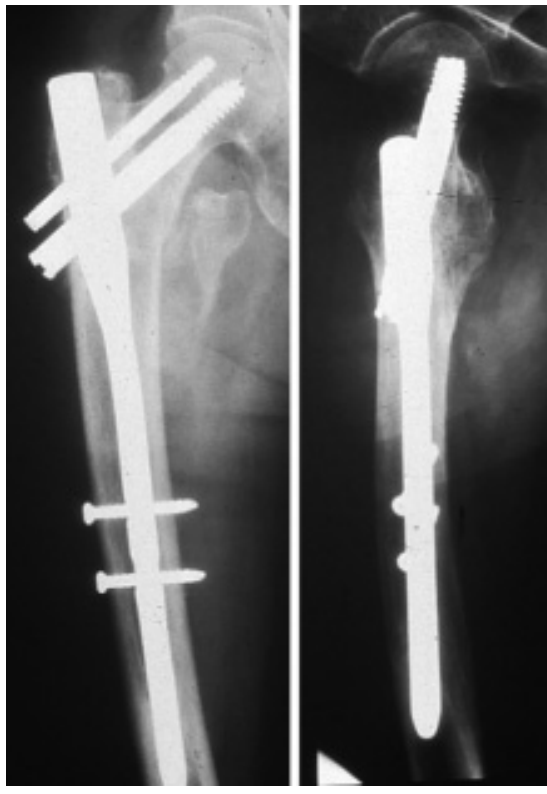

Abb. 8 Proximaler Femurmarknagel als klassisches Beispiel eines winkelstabilen, schräg axialen dynamischen Systems.

\section{Sonstige Systeme}

Im weitesten Sinn des Wortes müssen auch „Stapler und Krampen“ wie wir sie bei der Fußchirugie und zum einseitigen Wachstumsstop bei Epiphysen finden zu den winkelsteifen Implantaten gerechnet werden. Die Winkelstabilität kann hier noch mit Federspannung und/oder Memoryeffekt kombiniert werden. Auch diese Systeme werden in Zukunft weitere technologische Innovationen erfahren, welche noch ungelöste oder schwer zu behandelnde Spezialprobleme zu lösen helfen werden.

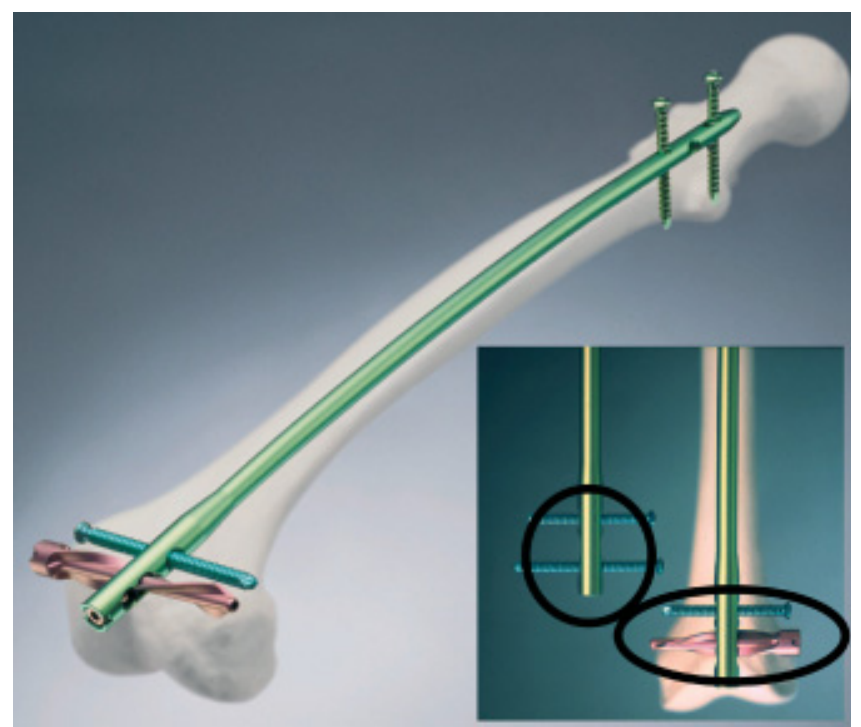

Abb. 9 Distaler Femur Nagel der AO mit winkelstabiler Spiralklinge. 


\section{Schlussbemerkung}

Die winkelsteifen Systeme haben bei genauer Betrachtung schon in der Vergangenheit große Dienste erwiesen. In erster Linie sei hier noch einmal an die Winkelplatten erinnert, welche neben der Osteosynthese der ersten Wahl auch häufig Rettungsanker sind. Die neue Form der Winkelstabilität ist in den internen Fixateursystemen verwirklicht. Biomechanische Vorteile und neue Formen der Implantation bis hin zu minimalinvasiven Methoden finden hier eine günstige Synthese, welche bereits heute und besonders in der Zukunft ganz neue Osteosyntheseformen ermöglichen.

\section{Literatur}

1 Müller M, Allgöwer M, Schneider R, Willenegger H. Manual of Internal Fixation. Springer, Berlin 1992

2 Rüedi TP, et al. AO Prinzipien des Frakturmanagements. Thieme, Stuttgart 2003
3 Synthes Hauptkataloge 2000-2003

${ }^{4}$ Wolter D, Hansis M, Havemann D. Externe und interne Fixateursysteme. Springer, Berlin 1995

Prof. Dr. med. Dankward Höntzsch Leitender Arzt

BG-Unfallklinik Tübingen

Schnarrenbergstr. 95

D-72076 Tübingen

\section{Buchbesprechung}

\section{Rüter A, Trentz 0, Wagner M. Unfallchirurgie. Urban \& Fischer, München, 2. Auflage 2004, € 199.-, ISBN 3-437-21850-6}

Nach fast neun Jahren erscheint nun die 2., komplett überarbeitete und erweiterte Auflage mit 1310 Seiten und 1000 s/w sowie 493 farbigen Abbildungen. Das „VielMänner-Buch“ repräsentiert inhaltlich überwiegend drei den Prinzipien der AO nahe stehende unfallchirurgische Schulen (Ulm/Hannover/Wien). Die bewährte Gliederung in einen allgemeinen und einen speziellen Teil wurde beibehal- ten. In der jetzt vorliegenden Auflage wurden neu aufgenommen die Intensivbehandlung und Anästhesie des Schwerverletzten, die Therapie offener und geschlossener Frakturen mit Weichteilschaden, Schusswunden und Explosionstrauma, Grundzüge der Antibiotikatherapie und -prophylaxe, Prinzipien der gedeckten Reposition und der konservativen Frakturbehandlung, Amputation und Prothetik, Akutmaßnahmen bei Augenund HNO-Verletzungen sowie die Darstellung der Schulterverletzungen in vier Teilkapiteln. Inhaltlich versucht das Buch der raschen Entwicklung der opera- tiven und technischen Möglichkeiten Rechnung $\mathrm{zu}$ tragen. Es ist gelungen, die gesamte Unfallchirurgie mit perioperativem Management sowie Begutachtung in einem Band zu vereinen. Etwas aktuellere Literaturzitate und mehr praktische Tipps können eine spätere Neuauflage noch abrunden. Mit einem mehrbändigen Werk kann und will sich dieses Buch nicht vergleichen. Es bietet dem Chirurgen in Weiterbildung alles für eine aktuelle Orientierung zu einem angemessenen Preis.

Karl Heinrich Winker 